\title{
Additivity in minimum cost spanning tree problems*
}

\author{
Gustavo Bergantiños \\ Research Group in Economic Analysis. Universidade de Vigo \\ Juan Vidal-Puga ${ }^{\dagger}$ \\ Research Group in Economic Analysis. Universidade de Vigo
}

March 24, 2008

\begin{abstract}
We characterize a rule in minimum cost spanning tree problems using an additivity property and some basic properties. If the set of possible agents has at least three agents, these basic properties are symmetry and separability. If the set of possible agents has two agents, we must add positivity.
\end{abstract}

JEL Codes: C71, D70, D85.

Keywords: minimum cost spanning tree problems, additivity.

\section{Introduction}

Imagine that a group of agents, located at different geographical places, want some particular service which can only be provided by a common supplier, called the source. Agents will be served through connections which entail some cost. However, they do not care whether they are connected directly or indirectly to the source. This situation is described by a symmetric matrix $C$, which specifies the connection costs between each pair agent-agent and agent-source. There are many situations that can be modeled in this way. For instance, several towns may draw power from a common power plant,

*Financial support by the Spanish Ministerio de Ciencia y Tecnología and FEDER through grant SEJ2005-07637-C02-01/ECON, and the Xunta de Galicia through grants PGIDIT06PXIC300184PN and PGIDIT06PXIB362390PR, is gratefully acknowledged.

${ }^{\dagger}$ Corresponding author. Address: Facultade de Ciencias Sociais. Campus A Xunqueira. 36005 Pontevedra. Spain. Phone: +34 986 802014. Fax: +34 986 812401. E-mail: vidalpuga@uvigo.es 
and hence have to share the cost of the distribution network (Dutta and Kar, 2004). Bergantiños and Lorenzo $(2004,2005)$ study a real situation where villagers had to pay the cost of constructing pipes from their respective houses to a water supplier. Other examples include communication networks, such as telephone, Internet, or cable television.

We assume that the agents construct a minimum cost spanning tree $(m t)$. The question is how to divide the cost associated with the $m t$ between the agents. A rule determines an allocation.

In this paper, we characterize a rule using a property of additivity. In its most natural formulation in mcstp, additivity is very demanding and no rule satisfies it. Hence, we introduce a restricted additivity. Other properties used in the characterization are symmetry, positivity, and separability. Symmetry says that symmetric agents must pay the same. Positivity says that every agent must pay at least zero.

Separability appears in Megiddo (1978), Granot and Huberman (1981), and Granot and Maschler (1998) with the name of decomposition, and in Bergantiños and VidalPuga (2007) with the name of separability. Two subsets of agents can connect to the source separately or jointly. If there are no savings when they connect jointly, separability says that the agents must pay the same in both circumstances.

Our results are the following. If the set of possible agents has at least three members, then there is a unique rule satisfying restricted additivity, symmetry, and separability. If the set of possible agents has exactly two members, then there is a unique rule satisfying positivity, restricted additivity, symmetry, and separability.

The rule we obtain in these characterizations is well known in the literature of mcstp. This rule was first introduced by Feltkamp, Tijs, and Muto (1994) and studied later by Brânzei, Moretti, Norde, and Tijs (2004) and Bergantiños and Vidal-Puga (2004, 2006, 2007).

Our paper is very related to the paper of Brânzei et al (2004) because they also characterize this rule using an additivity property and other two properties: equal treatment and upper bound contributions. These properties are very related to the concept of $C$-component. A $C$-component is a maximal coalition of agents that can be connected among themselves at zero cost. A rule satisfies equal treatment if all the members of a $C$-component receive the same. A rule satisfies upper bound contributions if the aggregate allocation assigned to the members of a $C$-component is not more than their connection cost to the source.

In Section 2 we introduce the model. In Section 3 we present our results. 


\section{Minimum cost spanning tree problems}

Let $\mathcal{N}=\{1,2, \ldots\}$ be the set of all possible agents. Given a finite set $N \subset \mathcal{N}$, let $\Pi_{N}$ be the set of all permutations over $N$. Given $\pi \in \Pi_{N}$, let $\pi_{p}$ denote the agent at position $p \in\{1, \ldots,|N|\}$ in the order $\pi$.

We are interested in networks whose nodes are elements of a set $N_{0}=N \cup\{0\}$, where $N \subset \mathcal{N}$ is finite and 0 is a special node called the source.

A cost matrix $C=\left(c_{i j}\right)_{i, j \in N_{0}}$ on $N$ represents the cost of direct link between any pair of nodes. We assume that $c_{i j}=c_{j i} \geq 0$ for each $i, j \in N_{0}$ and $c_{i i}=0$ for each $i \in N_{0}$. Since $c_{i j}=c_{j i}$ we work with undirected arcs, i.e. $(i, j)=(j, i)$.

We denote the set of all cost matrices over $N$ as $\mathcal{C}^{N}$.

A minimum cost spanning tree problem, briefly a mcstp, is a pair $\left(N_{0}, C\right)$ where $N \subset \mathcal{N}$ is a finite set of agents, 0 is the source, and $C \in \mathcal{C}^{N}$ is the cost matrix. Given a mcstp $\left(N_{0}, C\right)$, we denote the mcstp induced by $C$ in $S \subset N$ as $\left(S_{0}, C\right)$.

A network $g$ over $N_{0}$ is a subset of $\left\{(i, j): i, j \in N_{0}\right\}$. The elements of $g$ are called arcs. Given a network $g$ and a pair of nodes $i$ and $j$, a path from $i$ to $j$ in $g$ is a sequence of different arcs $\left\{\left(i_{h-1}, i_{h}\right)\right\}_{h=1}^{l}$ satisfying $\left(i_{h-1}, i_{h}\right) \in g$ for all $h \in\{1,2, \ldots, l\}, i=i_{0}$, and $j=i_{l}$. A tree is a network such that for all $i \in N$ there is a unique path from $i$ to the source. If $t$ is a tree, we usually write $t=\left\{\left(i^{0}, i\right)\right\}_{i \in N}$ where $i^{0}$ represents the first agent in the unique path in $t$ from $i$ to 0 .

Let $\mathcal{G}^{N}$ denote the set of all networks over $N_{0}$. Let $\mathcal{G}_{0}^{N}$ denote the set of all networks where every agent $i \in N$ is connected to the source, i.e. there exists a path from $i$ to 0 in the network.

Given a mcstp $\left(N_{0}, C\right)$ and $g \in \mathcal{G}^{N}$, we define the cost associated with $g$ as $c\left(N_{0}, C, g\right)=\sum_{(i, j) \in g} c_{i j}$. When there is no ambiguity, we write $c(g)$ or $c(C, g)$ instead of $c\left(N_{0}, C, g\right)$.

A minimum cost spanning tree for $\left(N_{0}, C\right)$, briefly an $m t$, is a tree $t$ over $N_{0}$ such that $c(t)=\min _{g \in \mathcal{G}_{0}^{N}} c(g)$. It is well-known that an $m t$ exists, even though it is not necessarily unique. Given a mcstp $\left(N_{0}, C\right)$, we denote the cost associated with any $m t$ as $m\left(N_{0}, C\right)$.

A (cost allocation) rule is a function $\psi$ that assigns to each mcstp $\left(N_{0}, C\right)$ a vector $\psi\left(N_{0}, C\right) \in \mathbb{R}^{N}$ such that $\sum_{i \in N} \psi_{i}\left(N_{0}, C\right)=m\left(N_{0}, C\right)$.

A coalitional game with transferable utility, briefly a $T U$ game, is a pair $(N, v)$ where $v: 2^{N} \rightarrow \mathbb{R}$ satisfies $v(\varnothing)=0 . S h(N, v)$ denotes the Shapley value (Shapley, $1953)$ of $(N, v)$. It is possible to associate a $T U$ game $\left(N, v_{C}\right)$ with each mcstp $\left(N_{0}, C\right)$ 
as follows. For each $S \subset N, v_{C}(S)=m\left(S_{0}, C\right)$.

In this paper we focus on a rule first introduced by Feltkamp et al (1994), which we denote as $\varphi$. This rule can be defined as

$$
\varphi\left(N_{0}, C\right)=S h\left(N, v_{C^{*}}\right)
$$

where $C^{*}$ is the irreducible matrix. For all $i, j \in N_{0}, c_{i j}^{*}=\max _{(k, j) \in g_{i j}}\left\{c_{k l}\right\}$ where $g_{i j}$ is the unique path connecting $i$ and $j$ in an $m t t$. See Bergantiños and Vidal-Puga (2007) for more details.

Finally, we define $C$-components following Norde, Moretti, and Tijs (2004). This concept will be used in some of the proofs. Given a mcstp $\left(N_{0}, C\right)$ and $S \subset N_{0}$, we say that $i, j \in N_{0}, i \neq j$ are $(C, S)$-connected if there exists a path $g$ from $i$ to $j$ satisfying that $g \in \mathcal{G}^{S}$ and $c_{k l}=0$ for all $(k, l) \in g$. We say that $S \subset N_{0}$ is a $C$-component if two conditions hold. First, for all $i, j \in S, i$ and $j$ are $(C, S)$-connected. Second, $S$ is maximal, i.e. if $S \varsubsetneqq T$ there exist $i, j \in T, i \neq j$ such that $i$ and $j$ are not $(C, T)$-connected.

Clearly, the set of $C$-components is a partition of $N_{0}$.

\section{The axiomatic characterization}

In its most natural definition, we say that a rule $\psi$ satisfies additivity if for all mcstp $\left(N_{0}, C\right)$ and $\left(N_{0}, C^{\prime}\right), \psi\left(N_{0}, C+C^{\prime}\right)=\psi\left(N_{0}, C\right)+\psi\left(N_{0}, C^{\prime}\right)$. However, no rule satisfies this property. Take

$C=\left(\begin{array}{ccc}0 & 10 & 50 \\ 10 & 0 & 10 \\ 50 & 10 & 0\end{array}\right), C^{\prime}=\left(\begin{array}{ccc}0 & 50 & 10 \\ 50 & 0 & 10 \\ 10 & 10 & 0\end{array}\right)$, and $C+C^{\prime}=\left(\begin{array}{ccc}0 & 60 & 60 \\ 60 & 0 & 20 \\ 60 & 20 & 0\end{array}\right)$.

Notice that $m\left(N_{0}, C\right)=m\left(N_{0}, C^{\prime}\right)=20$ and $m\left(N_{0}, C+C^{\prime}\right)=80$. By definition, $\sum_{i \in N} \psi_{i}\left(N_{0}, C\right)=m\left(N_{0}, C\right)$ for all mcstp $\left(N_{0}, C\right)$. Then, $\psi\left(N_{0}, C+C^{\prime}\right)=\psi\left(N_{0}, C\right)+$ $\psi\left(N_{0}, C^{\prime}\right)$ is not possible.

Hence, we should only claim additivity when the pair of problems are "similar", and not in any case. Moreover, we also want to claim additivity in a large subclass of problems.

In mcstp there exists an additivity property called cone-wise positive linearity $(C P L)$, which has been introduced by Brânzei et al (2004). We say that $\psi$ satisfies $C P L$ if $\psi\left(N_{0}, C+C^{\prime}\right)=\psi\left(N_{0}, C\right)+\psi\left(N_{0}, C^{\prime}\right)$ for all mcstp $\left(N_{0}, C\right)$ and $\left(N_{0}, C^{\prime}\right)$ 
satisfying that there exists an order for the $\operatorname{arcs} \sigma:\{(i, j)\}_{i, j \in N_{0}, i<j} \rightarrow\left\{1,2, \ldots ., \frac{n(n+1)}{2}\right\}$ such that if $i, j, k, l \in N_{0}$ with $i<j, k<l$, and $\sigma(i, j) \leq \sigma(k, l)$, then $c_{i j} \leq c_{k l}$ and $c_{i j}^{\prime} \leq c_{k l}^{\prime}$.

Notice that according to this definition ${ }^{1}$ two problems are "similar" when, ordering the arcs by their cost, we can obtain the same order in $C$ and $C^{\prime}$.

We now introduce our additivity property. If we want to claim $\psi\left(N_{0}, C+C^{\prime}\right)=$ $\psi\left(N_{0}, C\right)+\psi\left(N_{0}, C^{\prime}\right)$ we need, at least, that $m\left(N_{0}, C+C^{\prime}\right)=m\left(N_{0}, C\right)+m\left(N_{0}, C^{\prime}\right)$.

Let $t$ be an $m t$ in $\left(N_{0}, C+C^{\prime}\right)$. It is easy to see that $t$ is an $m t$ in both $\left(N_{0}, C\right)$ and $\left(N_{0}, C^{\prime}\right)$. Assume that we order the arcs in $t$ in non-decreasing cost. If we obtain the same order in $\left(N_{0}, C\right)$ and $\left(N_{0}, C^{\prime}\right)$, then we claim additivity on these problems. This is our idea of "similar" problems.

Formally, we say that $\psi$ satisfies restricted additivity $(R A)$ if

$$
\psi\left(N_{0}, C+C^{\prime}\right)=\psi\left(N_{0}, C\right)+\psi\left(N_{0}, C^{\prime}\right)
$$

for all mcstp $\left(N_{0}, C\right)$ and $\left(N_{0}, C^{\prime}\right)$ satisfying that there exists an $m t t=\left\{\left(i^{0}, i\right)\right\}_{i \in N}$ in $\left(N_{0}, C\right),\left(N_{0}, C^{\prime}\right)$, and $\left(N_{0}, C+C^{\prime}\right)$ and an order $\pi \in \Pi_{N}$ such that $c_{\pi_{1}^{0} \pi_{1}} \leq c_{\pi_{2}^{0} \pi_{2}} \leq$ $\ldots \leq c_{\pi_{n}^{0} \pi_{n}}$ and $c_{\pi_{1}^{0} \pi_{1}}^{\prime} \leq c_{\pi_{2}^{0} \pi_{2}}^{\prime} \leq \ldots \leq c_{\pi_{n}^{0} \pi_{n}}^{\prime}$.

It is straightforward to check that $R A$ implies $C P L$.

We now introduce the "basic" properties we use in our characterization results.

Given an mcstp $\left(N_{0}, C\right)$, we say that $i, j \in N, i \neq j$ are symmetric if for all $k \in N_{0} \backslash\{i, j\}, c_{i k}=c_{j k}$.

We say that $\psi$ satisfies symmetry $(S Y M)$ if for all mcstp $\left(N_{0}, C\right)$ and all pair of symmetric agents $i, j \in N, \psi_{i}\left(N_{0}, C\right)=\psi_{j}\left(N_{0}, C\right)$.

We say that $\psi$ satisfies positivity $(P O S)$ if for all mcstp $\left(N_{0}, C\right)$ and all $i \in N$, $\psi_{i}\left(N_{0}, C\right) \geq 0$.

We say that $\psi$ satisfies separability $(S E P)$ if for all mcstp $\left(N_{0}, C\right)$ and $S \subset N$ satisfying $m\left(N_{0}, C\right)=m\left(S_{0}, C\right)+m\left((N \backslash S)_{0}, C\right)$,

$$
\psi_{i}\left(N_{0}, C\right)= \begin{cases}\psi_{i}\left(S_{0}, C\right) & \text { if } i \in S \\ \psi_{i}\left((N \backslash S)_{0}, C\right) & \text { if } i \in N \backslash S\end{cases}
$$

for all $i \in N$.

\footnotetext{
${ }^{1}$ Brânzei et al (2004) define $C P L$ in a little bit different way. Take $x, x^{\prime} \geq 0$. They say that a rule $\psi$ satisfies $C P L$ if $\psi\left(N_{0}, x C+x^{\prime} C^{\prime}\right)=x \psi\left(N_{0}, C\right)+x^{\prime} \psi\left(N_{0}, C^{\prime}\right)$ when $\left(N_{0}, C\right)$ and $\left(N_{0}, C^{\prime}\right)$ are "similar". It is not difficult to check that the characterization of Brânzei et al (2004) also holds with our definition. We present it in a different way only because it is simpler.
} 
Two subsets of the agents, $S$ and $N \backslash S$, can connect to the source separately or can connect jointly. If there are no savings when they connect jointly, $S E P$ says that agents must pay the same in both circumstances.

Proposition 1. $\varphi$ satisfies $S E P, S Y M$, and $R A$.

Proof. In Bergantiños and Vidal-Puga (2007), we proved that $\varphi$ satisfies SEP. On the other hand, $\varphi$ is the Shapley value of an associated game. From this, it is straightforward to prove that $\varphi$ satisfies $S Y M$.

We now prove that $\varphi$ satisfies $R A$. Let $\left(N_{0}, C\right)$ and $\left(N_{0}, C^{\prime}\right)$ be two mcstp and let $t=\left\{\left(i^{0}, i\right)\right\}_{i \in N}$ be an $m t$ in $\left(N_{0}, C\right),\left(N_{0}, C^{\prime}\right)$, and $\left(N_{0}, C+C^{\prime}\right)$ satisfying that there exists an order $\pi \in \Pi_{N}$ such that $c_{\pi_{1}^{0} \pi_{1}} \leq c_{\pi_{2}^{0} \pi_{2}} \leq \ldots \leq c_{\pi_{n}^{0} \pi_{n}}$ and $c_{\pi_{1}^{0} \pi_{1}}^{\prime} \leq c_{\pi_{2}^{0} \pi_{2}}^{\prime} \leq \ldots \leq$ $c_{\pi_{n}^{0} \pi_{n}}^{\prime}$.

For all $i, j \in N_{0}, c_{i j}^{*}=\max _{(k, l) \in g_{i j}}\left\{c_{k l}\right\}, c_{i j}^{\prime *}=\max _{(k, l) \in g_{i j}}\left\{c_{k l}^{\prime}\right\}$, and $\left(c+c^{\prime}\right)_{i j}^{*}=\max _{(k, l) \in g_{i j}}\left\{c_{k l}+c_{k l}^{\prime}\right\}$ where $g_{i j}$ is the (unique) path in $t$ connecting $i$ and $j$. Hence, $\left(C+C^{\prime}\right)^{*}=C^{*}+C^{* *}$.

Applying Proposition 2.3 in Bergantiños and Vidal-Puga (2007), it is not difficult to deduce that for all $S \subset N, v_{\left(C+C^{\prime}\right)^{*}}(S)=v_{C^{*}}(S)+v_{C^{\prime *}}(S)$. Thus, the result follows from the additivity of the Shapley value.

Assume that $\mathcal{N}$ has at least three agents. Then we have the following result:

Proposition 2. There is a unique rule satisfying $S E P, S Y M$, and $R A$.

Proof. From Theorem 1 in Norde et al (2004), if $\left(N_{0}, C\right)$ is a mcstp, then there exists a family $\left\{C^{p}\right\}_{p=1}^{a}$ of cost matrices satisfying three conditions:

1. $C=\sum_{p=1}^{a} C^{p}$.

2. For each $p \in\{1, \ldots, a\}$ there exist $x^{p} \in \mathbb{R}$ and a network $g^{p}$ such that $c_{i j}^{p}=x_{p}$ if $(i, j) \in g^{p}$ and $c_{i j}^{p}=0$ otherwise.

3. There exists $\sigma:\{(i, j)\}_{i, j \in N_{0}, i<j} \rightarrow\left\{1,2, \ldots, \frac{n(n+1)}{2}\right\}$ such that if $i, j, k, l \in N$ with $i<j, k<l$, and $\sigma(i, j) \leq \sigma(k, l)$, then $c_{i j} \leq c_{k l}$ and $c_{i j}^{p} \leq c_{k l}^{p}$ for all $p \in\{1, \ldots, a\}$.

Let $\psi$ be a rule satisfying $S Y M, S E P$, and $R A$. Since $R A$ implies $C P L$, from conditions 1 and $3, \psi\left(N_{0}, C\right)=\sum_{p=1}^{a} \psi\left(N_{0}, C^{p}\right)$. 
Hence, it is enough to prove that $\psi$ is unique on the subclass of mcstp $\left(N_{0}, C\right)$ satisfying that there exist $x \in \mathbb{R}$ and a network $g$ such that $c_{i j}=x$ if $(i, j) \in g$ and $c_{i j}=0$ otherwise. Assume that $\left(N_{0}, C\right)$ satisfies these conditions.

Let $\left\{\left(N_{1}\right)_{0}, N_{2}, \ldots, N_{q}\right\}$ be the partition of $N_{0}$ in $C$-components. It is straightforward to check that $m\left(N_{0}, C\right)=\sum_{r=1}^{q} m\left(\left(N_{r}\right)_{0}, C\right)$. Under $S E P, \psi_{i}\left(N_{0}, C\right)=$ $\psi_{i}\left(\left(N_{r}\right)_{0}, C\right)$ for all $r \in\{1, \ldots, q\}$ and $i \in N_{r}$.

Hence, we can assume that all the agents can be connected among themselves at zero cost. This implies that, given $t=\left\{\left(i^{0}, i\right)\right\}$ is an $m t$ in $\left(N_{0}, C\right)$, there exists $\alpha \in N$ such that $\alpha^{0}=0$ and $c_{i{ }^{0} i}=0$ for all $i \in N \backslash\{\alpha\}$. We define $C^{1}$ and $C^{2}$ as follows:

$$
c_{i j}^{1}=\left\{\begin{array}{ll}
c_{i j} & \text { if } 0 \in\{i, j\} \\
0 & \text { otherwise }
\end{array} \quad \text { and } c_{i j}^{2}= \begin{cases}0 & \text { if } 0 \in\{i, j\} \\
c_{i j} & \text { otherwise }\end{cases}\right.
$$

It is not difficult to see that $t$ is an $m t$ in both $\left(N_{0}, C^{1}\right)$ and $\left(N_{0}, C^{2}\right)$. Moreover, $c_{i^{0} i}^{1}=c_{i^{0} i}^{2}=0$ for all $i \in N \backslash\{\alpha\}, c_{0 \alpha}^{1} \geq 0=c_{0 \alpha}^{2}$, and $C=C^{1}+C^{2}$. Under $R A$, $\psi\left(N_{0}, C\right)=\psi\left(N_{0}, C^{1}\right)+\psi\left(N_{0}, C^{2}\right)$.

Since $m\left(\{i\}_{0}, C^{2}\right)=0$ for all $i \in N$ and $m\left(N_{0}, C\right)=0$, under $S E P, \psi_{i}\left(N_{0}, C^{2}\right)=$ $\psi_{i}\left(\{i\}_{0}, C^{2}\right)=0$ for all $i \in N$. Hence, $\psi\left(N_{0}, C\right)=\psi\left(N_{0}, C^{1}\right)$.

Thus, it is enough to prove that $\psi$ is unique on the subclass of mcstp $\left(N_{0}, C\right)$ where $c_{i j}=0$ if $0 \notin\{i, j\}$ and $c_{0 i} \in\{0, x\}$ for all $i \in N$. If $c_{0 i}=0$ for all $i \in N$, or $c_{0 i}=x$ for all $i \in N$, all the agents are symmetric. Under $S Y M, \psi_{i}\left(N_{0}, C\right)$ is 0 or $\frac{x}{|N|}$ for all $i \in N$. If there exist $j, k \in N$ such that $c_{0 j}=x$ and $c_{0 k}=0$, we define $N^{1}=\left\{i \in N \mid c_{0 i}=x\right\} \cup\{k\}$ and $N^{2}=\left\{i \in N \mid c_{0 i}=0\right\} \backslash\{k\}$. These sets are under the conditions of SEP and hence $\psi_{i}\left(N_{0}, C\right)=\psi_{i}\left(N_{0}^{1}, C\right)$ for all $i \in N^{1}$ and $\psi_{i}\left(N_{0}, C\right)=\psi_{i}\left(N_{0}^{2}, C\right)$ for all $i \in N^{2}$. Notice that $\left(N_{0}^{2}, C\right)$ is in the same case as before.

Thus, it is enough to prove that $\psi$ is unique on the subclass of $\operatorname{mcstp}\left(N_{0}, C\right)$ where there exists $k \in N$ such that $c_{0 i}=x \in \mathbb{R}$ if $i \neq k$ and $c_{i j}=0$ otherwise.

For each $i \in N \backslash\{k\}$, let $\left(N, C^{i}\right)$ such that $c_{0 i}^{i}=x$ and $c_{j l}^{i}=0$ otherwise. Notice that $C=\sum_{i \in N \backslash\{k\}} C^{i}$. Moreover, $t=\{(j-1, j)\}_{j=2}^{n} \cup\{(0, k)\}$ is an $m t$ in $\left(N_{0}, C^{i}\right)$ for all $i \in N \backslash\{k\}$. Under $R A, \psi\left(N_{0}, C\right)=\sum_{i \in N \backslash\{k\}} \psi\left(N_{0}, C^{i}\right)$.

Thus, it is enough to prove that for all $i \in N \backslash\{k\}, \psi$ is unique in each problem $\left(N_{0}, C^{i}\right)$. Take $i \in N \backslash\{k\}$. It is trivial to see that

$$
m\left(N_{0}, C^{i}\right)=m\left(\{k, i\}_{0}, C^{i}\right)+\sum_{j \in N \backslash\{k, i\}} m\left(\{j\}_{0}, C^{i}\right) .
$$


Under SEP, $\psi_{k}\left(N_{0}, C^{i}\right)=\psi_{k}\left(\{k, i\}_{0}, C^{i}\right), \psi_{i}\left(N_{0}, C^{i}\right)=\psi_{i}\left(\{k, i\}_{0}, C^{i}\right)$, and for all $j \in N \backslash\{k, i\}, \psi_{j}\left(N_{0}, C^{i}\right)=\psi_{j}\left(\{j\}_{0}, C^{i}\right)=0$.

It only remains to prove that $\psi$ is unique in the $\operatorname{mcstp}(\{i, k\}, C)$ where $c_{0 k}=c_{i k}=$ 0 and $c_{0 i}=x$.

Since $m\left(\{i, k\}_{0}, C\right)=0, \psi\left(\{i, k\}_{0}, C\right)=(y,-y)$. We prove that $y=0$.

Let $\left(N_{0}, C^{\prime}\right)$ be such that $N=\{i, j, k\}, c_{0 i}^{\prime}=x$, and $c_{h l}^{\prime}=0$ otherwise. We can find such a $\left(N_{0}, C^{\prime}\right)$ because $\mathcal{N}$ has at least three members.

Hence, $m\left(N_{0}, C^{\prime}\right)=m\left(\{j\}_{0}, C^{\prime}\right)+m\left(\{i, k\}_{0}, C^{\prime}\right)$. Under $S E P$,

$$
\psi_{k}\left(N_{0}, C^{\prime}\right)=\psi_{k}\left(\{i, k\}_{0}, C^{\prime}\right)=\psi_{k}\left(\{i, k\}_{0}, C\right)=-y
$$

Moreover, $m\left(N_{0}, C^{\prime}\right)=m\left(\{i, j\}_{0}, C^{\prime}\right)+m\left(\{k\}_{0}, C^{\prime}\right)$. Under $S E P, \psi_{k}\left(N_{0}, C^{\prime}\right)=$ $\psi_{k}\left(\{k\}_{0}, C^{\prime}\right)=0$. Hence, $y=0$.

The next theorem is a trivial consequence of Propositions 1 and 2.

Theorem 1. If $\mathcal{N}$ has at least three agents, then $\varphi$ is the unique rule satisfying $S E P, S Y M$, and $R A$.

The properties used in Theorem 1 are independent.

Assume $N=\{1, \ldots, n\}$. For each $\operatorname{mcstp}\left(N_{0}, C\right)$ and $i \in N$ we define $\psi_{i}^{1}\left(N_{0}, C\right)=$ $v_{C^{*}}(\{1, \ldots, i\})-v_{C^{*}}(\{1, \ldots, i-1\}) . \psi^{1}$ satisfies $S E P$ and $R A$, but fails $S Y M$.

Consider the egalitarian rule defined as $\psi_{i}^{2}\left(N_{0}, C\right)=m\left(N_{0}, C\right) /|N|$ for all $i \in N$. $\psi^{2}$ satisfies $R A$ and $S Y M$, but fails $S E P$.

Let $\psi^{3}$ be defined as

$$
\psi_{i}^{3}\left(N_{0}, C\right)=\frac{1}{\left|\Pi_{N}^{\prime}\right|} \sum_{\pi \in \Pi_{N}^{\prime}}\left[v_{C^{*}}(\operatorname{Pre}(i, \pi) \cup\{i\})-v_{C^{*}}(\operatorname{Pre}(i, \pi))\right]
$$

for all $i \in N$, where $\Pi_{N}^{\prime}$ is the subset of orders in which the agents with the cheapest cost to the source connect first, i.e.

$$
\Pi_{N}^{\prime}=\left\{\pi \in \Pi_{N} \mid c_{0 \pi_{q}} \leq c_{0 \pi_{p}} \text { when } q<p\right\}
$$

$\psi^{3}$ satisfies $S E P$ (Bergantiños and Vidal-Puga, 2007) and $S Y M$, but fails $R A$.

The next theorem is the analogous to Theorem 1 when $\mathcal{N}$ has two agents.

Theorem 2. If $\mathcal{N}$ has two agents, then $\varphi$ is the unique rule satisfying $P O S, S E P$, $S Y M$, and $R A$. 
Proof. In Bergantiños and Vidal-Puga (2007), we proved that $\varphi$ satisfies POS. By Proposition 1 we know that $\varphi$ satisfies $S E P, S Y M$, and $R A$.

We now prove the uniqueness. Let $\psi$ be a rule satisfying POS, SYM, SEP , and $R A$. If $N=\{i\}$, by definition $\psi_{i}\left(N_{0}, C\right)=m\left(N_{0}, C\right)=c_{0 i}$.

Assume that $N=\{i, j\}$. Using arguments similar to those used in the proof of Proposition 2, we can deduce that it is enough to prove that $\psi$ is unique in the mcstp $\left(\{i, j\}_{0}, C\right)$ where $c_{0 i}=c_{i j}=0$ and $c_{0 j}=x$.

Since $m\left(N_{0}, C\right)=0, \psi_{i}\left(N_{0}, C\right)=-\psi_{j}\left(N_{0}, C\right)$. Under POS, $\psi_{i}\left(N_{0}, C\right)=\psi_{j}\left(N_{0}, C\right)=$ 0 .

The properties used in Theorem 2 are also independent. When $\mathcal{N}$ has two agents, $\psi^{1}$ satisfies $S E P, R A$ and $P O S$ but fails $S Y M ; \psi^{2}$ satisfies $S Y M, R A$ and $P O S$ but fails $S E P$; and $\psi^{3}$ satisfies $S E P, S Y M$ and $P O S$ but fails $R A$.

Assume, without loss of generality, that $N=\{i, j\}$ and $c_{0 i} \leq c_{0 j}$. We consider the rule $\psi^{4}$ defined as

$$
\begin{aligned}
& \psi_{i}^{4}\left(N_{0}, C\right)=\varphi_{i}\left(N_{0}, C\right)-\max \left\{0, c_{0 j}-\max \left\{c_{0 i}, c_{i j}\right\}\right\} \text { and } \\
& \psi_{j}^{4}\left(N_{0}, C\right)=\varphi_{j}\left(N_{0}, C\right)+\max \left\{0, c_{0 j}-\max \left\{c_{0 i}, c_{i j}\right\}\right\} .
\end{aligned}
$$

$\psi^{4}$ satisfies $S Y M, S E P$ and $R A$, but fails $P O S$.

In Bergantiños and Vidal-Puga (2007) we introduce, in mcstp, the property of Population Monotonicity $(P M)$. We say that $\psi$ satisfies $P M$ if for all mcstp $\left(N_{0}, C\right)$, all $S \subset N$, and all $i \in S, \psi_{i}\left(N_{0}, C\right) \leq \psi_{i}\left(S_{0}, C\right)$. $P M$ says that, if new agents join a society, no agent of the initial society can be worse off. Since $P M$ implies $S E P$ and $\varphi$ satisfies $P M$, all the results of this paper hold with $P M$ instead of $S E P$.

\section{References}

[1] Bergantiños G. and Lorenzo L. (2004) A non-cooperative approach to the cost spanning tree problem. Mathematical Methods of Operations Research 59, 393403.

[2] Bergantiños G. and Lorenzo L. (2005). Optimal equilibria in the non-cooperative game associated with cost spanning tree problems. Annals of Operations Research 137, 101-115. 
[3] Bergantiños G. and Vidal-Puga J. (2004) Several approaches to the same rule in cost spanning tree problems. Mimeo. Avaliable at http://webs.uvigo.es/vidalpuga

[4] Bergantiños G. and Vidal-Puga J. (2007) The optimistic TU game in minimum cost spanning tree problems. International Journal of Game Theory 36(2), 223-239, doi: $10.1007 / \mathrm{s} 00182-006-0069-7$.

[5] Bergantiños G. and Vidal-Puga J. (2007) A fair rule in minimum cost spanning tree problems. Journal of Economic Theory 137(1), 326-352, doi: 10.1016/j.jet.2006.11.001.

[6] Brânzei R., Moretti S., Norde H. and Tijs S. (2004) The P-value for cost sharing in minimum cost spanning tree situations. Theory and Decision 56, 47-61.

[7] Dutta B. and Kar A. (2004) Cost monotonicity, consistency and minimum cost spanning tree games. Games and Economic Behavior 48(2), 223-248.

[8] Feltkamp V., Tijs S. and Muto S. (1994) On the irreducible core and the equal remaining obligation rule of minimum cost extension problems. CentER DP 1994 nr.106, Tilburg University, The Netherlands.

[9] Granot D. and Huberman G. (1984) On the core and nucleolus of the minimum cost spanning tree games. Mathematical Programming 29, 323-347.

[10] Granot D. and Maschler M. (1998) Spanning network games. International Journal of Game Theory 27, 467-500.

[11] Megiddo N. (1978) Computational complexity and the game theory approach to cost allocation for a tree. Mathematics of Operations Research 3, 189-196.

[12] Norde H., Moretti S., and Tijs S. (2004) Minimum cost spanning tree games and population monotonic allocation schemes. European Journal of Operational Research 154, 84-97.

[13] Shapley L.S. (1953) A value for n-person games. Contributions to the Theory of Games II. Ed. by H.W. Kuhn and A.W. Tucker. Princeton NJ. Princeton University Press, 307-317. 